\title{
Oxidative Stress and Antioxidant Status in Fetal Circulation in Preeclampsia
}

\author{
KRISTIN BRAEKKE, NINA K. HARSEM, AND ANNE C. STAFF
}

\begin{abstract}
Department of Pediatric Intensive Care [K.B], Department of Obstetrics and Gynecology [N.K.H., A.C.S.], Ulleval University Hospital,
\end{abstract} 0407 Oslo, Norway

\begin{abstract}
Preeclampsia is associated with oxidative stress in maternal circulation. The purpose of this study was to explore oxidative stress and antioxidants in the fetal circulation in preeclampsia. Women with preeclampsia $(n=19)$ or uncomplicated pregnancies $(n=33)$ delivered by cesarean section were included. Blood was sampled separately from the umbilical vein and artery. 8-Isoprostaglandin $\mathrm{F}_{2 \alpha}$ (8-isoprostane), a stable product of lipid peroxidation, is a reliable marker of oxidative stress. Concentration of total 8 -isoprostane in cord plasma was analyzed by gas chromatographymass spectrometry. Antioxidant status was evaluated measuring ferric reducing ability of plasma and vitamin $\mathrm{E}$. There was no difference between preeclampsia and control groups regarding median plasma concentration of 8 -isoprostane in umbilical vein 955 versus 780 $\mathrm{pg} / \mathrm{mL}, p=0.41)$ or in umbilical artery (233 versus $276 \mathrm{pg} / \mathrm{mL}, p=$ $0.65)$. Concentration of 8 -isoprostane was much higher in plasma from the umbilical vein than artery, suggesting placenta as the source of 8-isoprostane. Median ferric reducing ability of plasma concentration was higher in preeclampsia than in controls, both in the umbilical vein and artery. Median vitamin E concentration in the umbilical vein was higher in preeclampsia, but no difference was found in the umbilical artery. In conclusion, no evidence of increased oxidative stress, evaluated by 8-isoprostane concentration, was found in fetal circulation in preeclampsia. (Pediatr Res 60: 560-564, 2006)
\end{abstract}

$\mathrm{P}_{\mathrm{b}}$ reeclampsia is a pregnancy specific disorder, characterized by hypertension and proteinuria developing in the second half of the pregnancy. For the fetus, the preeclampsia syndrome could have serious consequences, including increased risk of fetal growth restriction and induced premature delivery due to disease progression on the maternal or fetal side. Maternal endothelial dysfunction is believed to be the common final pathway leading to the syndrome of preeclampsia. A dominating hypothesis is that agents produced by the relatively hypoxic placenta is transferred to the maternal circulation, causing endothelial dysfunction (1). Several candidates have been proposed as possible agents that directly or indirectly could mediate the endothelial dysfunction, including lipid peroxidation products $(2,3)$, activated leukocytes (4), placental debris (5), or sFlt1, a vascular endothelial growth factor (VEGF) antagonist (6). To what extent such placental

Received December 16, 2005; accepted July 4, 2006.

Correspondence: Kristin Braekke, M.D., Department of Pediatric Intensive Care, Ulleval University Hospital, Kirkeveien 166, 0407 Oslo, Norway; e-mail: kbre@uus.no

Supported by the Norwegian Sudden Infant Death Association and the Norwegian Women's Public Health Association. agents affect the fetus, or are present in the fetal circulation at increased concentrations in preeclampsia, has not been fully explored. Few studies have addressed this issue, with conflicting results (7-10). If endothelial activating agents are present in the fetal circulation, they could cause immediate effects, such as endothelial activation, or even long-term consequences, such as the programming of adult diseases. The concept of intrauterine fetal programming, that an individual's adult health could be influenced by conditions and growth in utero, is convincingly documented in epidemiologic studies, including effects on blood pressure, cardiovascular risk factors and insulin resistance (11).

In studies of long-term effects of intrauterine exposure to preeclampsia, an increased risk of hypertension in the adolescent offspring $(12,13)$ as well as decreased risk of breast cancer have been reported (14), but the mechanisms are unknown (15).

Oxidative stress is defined as an imbalance between free radical damage and antioxidant protection. The F2-class of isoprostanes includes 8-isoprostane, which is a stable product of free radical-catalyzed peroxidation of arachidonic acid in cell membrane phospholipids (16). 8-Isoprostane is considered a highly reliable and stable marker of oxidative stress, and is increased in many diseases associated with oxidative stress, such as diabetes and atherosclerosis $(17,18)$. Increased oxidative stress has been proposed as a factor contributing to the endothelial dysfunction and pathophysiological changes in preeclampsia (3). Evidence for this concept includes studies demonstrating elevated levels of oxidative stress-markers in preeclampsia compared with normal pregnancies, in the placental tissue $(19,20)$, in the maternal circulation $(2,21)$, as well as in exhaled breath (22).

Presently, several clinical studies are assessing the effect of antioxidants, such as vitamin $\mathrm{C}$ and $\mathrm{E}$, in the prevention of preeclampsia (23), calling for further focus on the fetal situation in preeclamptic pregnancies. Premature infants, with their decreased antioxidant defense, are susceptible to oxidant injury and diseases associated with oxidative stress, such as bronchopulmonary dysplasia and retinopathy of prematurity

DOI: 10.1203/01.pdr.0000242299.01219.6a

Abbreviations: FRAP, ferric reducing ability of plasma; GC-MS, gas chromatography-mass spectrometry; 8-isoprostane, 8-iso-prostaglandin $\mathrm{F}_{2 \alpha}$; MDA, malondialdehyde; sFlt1, soluble fms-like tyrosine kinase 1 
(24-26). It is thus of interest to explore whether pregnancy complications such as preeclampsia add to the imbalance between pro- and antioxidants in the infant.

The aim of this study was to investigate the concentration of 8-isoprostane in plasma from the umbilical vein and artery of newborns from pregnancies complicated by preeclampsia, compared with uncomplicated pregnancies. We also wanted to evaluate the antioxidant system by measuring vitamin $\mathrm{E}$ and total antioxidants, measured as FRAP, in the same groups.

\section{METHODS}

Subjects. The included patients in this study were preeclamptic patients and women with uncomplicated pregnancies (called controls), all singleton pregnancies. The study is part of an ongoing biobank collection of patient samples from complicated and uncomplicated pregnancies at Ulleval University Hospital. The women included in the study were all delivered by cesarean section, all were fasting, none were in active labor and none had rupture of fetal membranes or signs of infection. Cesarean section was performed in the preeclamptic women $(n=19)$ because vaginal delivery was not considered appropriate due to disease progression and/or unfavorable cervical ripening. Controls $(n=33)$ were healthy, normotensive women undergoing cesarean section due to breech presentation, previous cesarean section or psychological reasons.

Preeclampsia was defined as rise in blood pressure after $20 \mathrm{wk}$ gestation to $>140 / 90$ on two or more occasions at least $6 \mathrm{~h}$ apart in a previously normotensive woman, combined with proteinuria. Proteinuria was defined as protein dip stick $\geq 1+$ on two or more midstream urine samples at least $6 \mathrm{~h}$ apart or a 24-h urine excretion of $\geq 0.3 \mathrm{~g}$ protein. Severe preeclampsia was defined according to the criteria of American College of Obstetricians and Gynecologists (ACOG) (27), including preeclamptic women with systolic blood pressure $\geq 160 \mathrm{~mm} \mathrm{Hg}$. Pregnancy duration was calculated on the basis of routine ultrasonographic screening between gestational wk 17 and 20. The newborn birth weight percentiles were calculated according to Norwegian birth registry data (28). Medical history and obstetrical information was obtained from hospital records and the patients' national pregnancy charts, as well as by interviewing the patients before cesarean delivery. The study protocol was approved by the Regional Committee of Medical Ethics in Norway, and informed written consent was obtained from each patient.

Biologic samples. During the delivery of the infant, the umbilical cord was double clamped both close to the infant and close to the placenta. Immediately after delivery of the placenta, blood from the umbilical arteries (transporting blood from the fetus to the placenta) and blood from the umbilical vein (transporting blood from the placenta to the fetus) was sampled separately into EDTA-containing vials for vitamin E and FRAP analyses, and into cold tubes containing $1 \mathrm{mg} / \mathrm{mL}$ citrate and indomethacin $15 \mu \mathrm{M}$ for the 8-isoprostane measurements. The blood samples were kept on ice for maximum $30 \mathrm{~min}$ before centrifugation for $10 \mathrm{~min}$ at $2000 \mathrm{~g}$ at $4^{\circ} \mathrm{C}$, and EDTA-plasma was stored at $-76^{\circ} \mathrm{C}$ until FRAP and vitamin $\mathrm{E}$ analysis. The plasma for the 8-isoprostane analysis was collected and protected from oxidation by addition of butylated hydroxytoluene (BHT) (Sigma Chemical Co., St. Louis, MO), to prevent ex vivo oxidation, at a final concentration of $20 \mu \mathrm{g} / \mathrm{mL}$ before storage at $-76^{\circ} \mathrm{C}$.

Total 8-isoprostane. Total 8-isoprostane concentration (free plus esterified 8-isoprostane) was measured by use of electron-capture negative-ion GC-MS by a collaborating laboratory in the United Kingdom, as previously described (29), after shipment on dry ice. All samples were measured in duplicates.
$\boldsymbol{F R A P}$. FRAP was measured in plasma with a photometer analyzer (Technicon Instruments Corporation, New York, NY), essentially as described $(30,31)$, with the exception that the sample was not diluted with water in the assay (21). All samples were measured in triplicates.

Vitamin E. A $50-\mu \mathrm{L}$ internal standard of $\alpha$-tocopherol was added to 500 $\mu \mathrm{L}$ of plasma. After addition of $0.5 \mathrm{~mL}$ water and $1 \mathrm{~mL}$ hexane, the solution was vortexed and centrifuged for $10 \mathrm{~min}$ at $4^{\circ} \mathrm{C}$. The organic phase was removed and dried under a nitrogen stream at $4^{\circ} \mathrm{C}$, and reconstituted in 100 $\mu \mathrm{L}$ acetonitrile and transferred to WISP auto-injector vials. Aliquots of $40 \mu \mathrm{L}$ were injected on to HPLC and detection of tocopherol was carried out using fluorescence (excitation wavelength $295 \mathrm{~nm}$ and emission wavelength 430 $\mathrm{nm})$. All samples were measured in duplicates.

Statistics. As all results were not normally distributed, medians are reported, and nonparametric Mann-Whitney $U$ test was used for testing differences between groups. Pearson's correlation was used to calculate correlation coefficients. Statistical analyses were performed using Statistical Package for the Social Sciences (version 11.0; SPSS Inc, Chicago, IL). A probability level of $\leq 0.05$ was considered statistically significant.

\section{RESULTS}

The clinical characteristics of the subjects included in the study are given in Table 1. Due to limited amount of fetal blood samples available, not all 19 included preeclamptic pregnancies described in Table 1 had all analyses performed.

For the preeclampsia group, median pregnancy duration was shorter and newborn weight was lower, but also the median weight percentile for infants born after preeclamptic pregnancies was lower than in the control group. In the control group, all infants were healthy and stayed in the maternity ward with their mothers after delivery. Five infants from preeclamptic pregnancies stayed in the maternity ward and 14 were transferred to the neonatal intensive care unit, as expected from their low gestational age ranging from 199 to $244 \mathrm{~d}$. In the intensive care unit, three of these infants required ventilator, another six needed continuous positive airway pressure, and three infants were given antibiotics due to invasive procedures, but there was no culture proven sepsis. All infants in the preeclampsia group (as well as in the control group) survived the neonatal period.

The individual concentrations of total 8-isoprostane in plasma from umbilical vein and artery for the two study groups are illustrated in Figure 1. The group values for umbilical vein and artery concentrations of 8-isoprostane, FRAP, and vitamin E are summarized in Table 2. We found no statistically significant difference between the preeclampsia and control groups regarding median concentration of total 8 -isoprostane in umbilical vein or in umbilical artery. As illustrated in Figure 1, there was a statistically significant

Table 1. Clinical characteristics for the patient groups included

\begin{tabular}{lccc}
\hline & Preeclampsia $(n=19)$ & Control $(n=33)$ & $p$ Value \\
\hline Maternal age $(\mathrm{y})$ & $31.5(19-39)$ & $32.5(22-38)$ & 0.39 \\
BMI before pregnancy $\left(\mathrm{kg} / \mathrm{m}^{2}\right)$ & $22.8(19.4-36.7)$ & $22.5(18.3-28.9)$ & 0.41 \\
BMI at delivery $\left(\mathrm{kg} / \mathrm{m}^{2}\right)$ & $28.6(22.5-39.5)$ & $29.7(23.6-37.5)$ & 0.44 \\
Gestational age $(\mathrm{d})$ & $235(199-271)$ & $271(259-283)$ & $<0.001^{*}$ \\
BP at delivery, systolic & $160(148-196)$ & $120(100-140)$ & $<0.001^{*}$ \\
BP at delivery, diastolic & $105(90-119)$ & $75(60-92)$ & $<0.001^{*}$ \\
Birth weight $(\mathrm{g})$ & $1719(870-3856)$ & $3472(2800-4680)$ & $<0.001^{*}$ \\
Birth weight percentile & $29(7-74)$ & $51(10-99)$ & $0.007 *$ \\
\hline
\end{tabular}

Values shown are medians and range (minimum and maximum values). Differences between the patient groups were tested using Mann-Whitney $U$ test. BMI, body mass index $\left(\mathrm{kg} / \mathrm{m}^{2}\right) ; \mathrm{BP}$, blood pressure.

$* p<0.05$. 


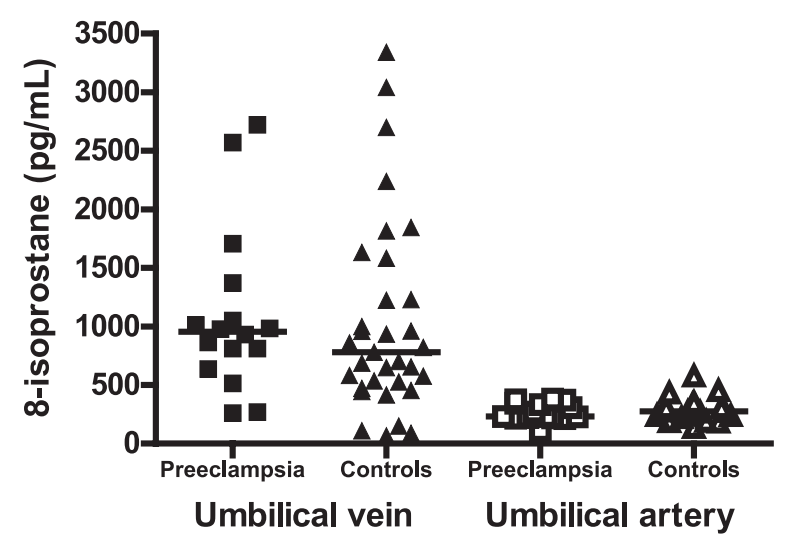

Figure 1. 8-Isoprostane concentrations $(\mathrm{pg} / \mathrm{mL})$ in umbilical vein and artery from preeclampsia $(n=19)$ and control $(n=33)$ pregnancies. Horizontal bars represent median values.

higher median concentration of 8-isoprostane in the umbilical vein compared with the umbilical artery for both pregnancy groups (preeclampsia group: umbilical vein $955 \mathrm{pg} / \mathrm{mL}$ versus umbilical artery $233 \mathrm{pg} / \mathrm{mL}, p=0.002$; control group: umbilical vein $780 \mathrm{pg} / \mathrm{mL}$ versus umbilical artery $276 \mathrm{pg} / \mathrm{mL}, p<$ $0.001)$. We found no correlation between gestational age and 8 -isoprostane concentration in the umbilical vein or artery for the whole group or for the preeclampsia group alone (data not shown). There was no correlation between FRAP and 8-isoprostane or between vitamin $\mathrm{E}$ and 8-isoprostane concentrations in the umbilical artery or vein (data not shown).

Median FRAP concentration was higher in the preeclampsia group than in the control group, both in the umbilical vein and artery. Median vitamin E concentration was higher in the preeclampsia group in the umbilical vein plasma compared with the control group, whereas no difference between the two study groups was found in the umbilical artery (Table 2).

There was no statistically significant difference between the subgroups of severe preeclampsia $(n=13)$ and moderate preeclampsia $(n=6)$ regarding fetal median 8-isoprostane, FRAP, or vitamin E concentrations, neither for the umbilical vein, nor for the umbilical artery (data not shown).

We found no statistically significant correlation between the concentrations of 8-isoprostane in umbilical vein and umbilical artery (Pearson $-0.12, p=0.50$ ). In contrast, the levels of FRAP in umbilical vein and artery correlated positively for the whole group (Pearson 0.83, $p<0.001$ ) and for the preeclampsia group separately (Pearson 0.97, $p<0.001$ ), and the same was found for vitamin $\mathrm{E}$ concentrations (for the whole group: Pearson $0.84, p<0.001$; for the preeclampsia group: Pearson $0.86, p=0.003)$.

\section{DISCUSSION}

In a previous publication by our group, we have reported the concentrations of the oxidative stress marker 8-isoprostane in the systemic circulation of the mothers of the newborns included in the present study (21). We reported that the preeclampsia group had a $62 \%$ higher median maternal plasma concentration of 8-isoprostane compared with the control group (354 and $218 \mathrm{pg} / \mathrm{mL}, p=0.02$ ). In the present study, we did not find that the increased maternal oxidative stress in preeclampsia was reflected in the fetal circulation; median 8-isoprostane concentration was not increased compared with control pregnancies, neither in umbilical vein nor artery. The much higher concentration of 8-isoprostane in umbilical vein plasma compared with umbilical artery plasma (Fig. 1), suggests that the placenta, and not the fetus, is the main source of fetal circulating 8-isoprostane. No correlation between previously published maternal (21) and fetal (neither umbilical vein nor artery) 8-isoprostane plasma concentration was found (neither for the whole patient group nor for the preeclampsia and control subgroups).

Few other studies have explored oxidative stress markers in the fetal circulation in preeclampsia. Kato et al. (8) reported a $26 \%$ higher mean concentration of malondialdehyde (MDA), another marker of oxidative stress, in preeclamptic pregnancies compared with controls, samples taken from umbilical vein at cordocentesis unaffected by labor. In contrast, Bowen et al. (7) found no difference between preeclampsia and control concentrations of MDA in umbilical vein. Recently pentosidine, an advanced glycation end product, which can also be used as a marker of oxidative stress, was explored in fetal circulation in preeclampsia (10). Mean pentosidine concentration was found to be $45 \%$ higher in blood from umbilical vein at delivery from infants born after preeclamptic pregnancies compared with controls, supporting a concept of elevated oxidative stress in the fetal circulation in preeclampsia.

To our knowledge, ours is the first study exploring oxidative stress in plasma separately from the umbilical vein and artery in preeclampsia. Other studies have used samples from the umbilical vein, which possibly does not give the most representative estimate of the fetal situation. Also, 8-isoprostane is considered a superior marker of oxidative stress. Other oxidized lipid products, such as MDA, represent more unstable products of lipid peroxidation, less specific and more likely to undergo auto-oxidation ex vivo (32).

Most studies of antioxidants in newborns report that concentrations of antioxidants are low, and even lower in premature infants $(24,26,33)$. The fetal concentration of lipid soluble

Table 2. 8-Isoprostane, FRAP, and vitamin E concentrations in umbilical vein and artery

\begin{tabular}{|c|c|c|c|}
\hline & Preeclampsia & Control & $p$ Value \\
\hline 8-Isoprostane $(\mathrm{pg} / \mathrm{mL})$ umbilical vein plasma & $955(636-1050)(n=16)$ & $780(576-1000)(n=33)$ & 0.41 \\
\hline 8-Isoprostane $(\mathrm{pg} / \mathrm{mL})$ umbilical artery plasma & $233(221-346)(n=15)$ & $276(238-320)(n=21)$ & 0.66 \\
\hline FRAP $(\mu \mathrm{mol} / \mathrm{L})$ umbilical vein plasma & $1101(913-1191)(n=14)$ & $899(807-966)(n=31)$ & $0.001 *$ \\
\hline Vitamin E ( $\mu \mathrm{mol} / \mathrm{L})$ umbilical vein plasma & $5.7(3.6-7.1)(n=14)$ & $3.6(3.3-4.5)(n=31)$ & $0.001 *$ \\
\hline Vitamin E ( $\mu \mathrm{mol} / \mathrm{L})$ umbilical artery plasma & $4.7(3.3-8.8)(n=10)$ & $3.7(2.6-4.9)(n=13)$ & 0.19 \\
\hline
\end{tabular}

Results are shown as medians (and 95\% confidence interval of medians). Differences between the patient groups were tested using Mann-Whitney $U$ test. $* p<0.05$. 
antioxidants is probably limited by placental transport proteins, and these antioxidants are found in lower concentrations in fetal than maternal circulation $(33,34)$. Vitamin E scavenges lipid soluble peroxide radicals and is the most important lipid soluble, chain-breaking antioxidant. It is consumed during peroxidation, and increased lipid peroxidation in preeclampsia could result in decreased plasma levels of vitamin E, described by some $(35,36)$, but not all authors (37) in the maternal circulation in preeclampsia. In our previously published study of the mothers of the newborns reported in the present study, we did not find any difference in maternal vitamin $\mathrm{E}$ concentrations between preeclampsia and controls (21). In preeclampsia, the fetal antioxidant status is largely unexplored. Lower maternal levels of carotenoids was found in preeclamptic women, whereas no differences were found between preeclampsia and controls in their infants' umbilical vein cord blood (38); cord values were about one tenth of maternal values. We found one publication on umbilical vein vitamin $\mathrm{E}$ levels in preeclampsia, reporting no difference between preeclampsia and controls (7). In contrast, we found in the present study significantly higher concentrations of vitamin $\mathrm{E}$ in umbilical vein plasma in preeclampsia compared with controls (Table 2). In the umbilical artery, which might more correctly reflect the situation in the fetus, transporting blood from the fetus to the placenta, we found no statistically significant difference in vitamin $\mathrm{E}$ concentrations between the groups. We found that the median concentrations in the umbilical artery were about $10 \%$ of our previously reported median maternal values [38.5 $\mu \mathrm{M}$ for both preeclampsia and control groups (21)]. Vitamin E concentration could be depending on the total lipid concentration (34), which might be higher in cord plasma in preeclampsia. Due to limited cord blood sample volumes, we have not measured total infant lipid concentrations in this study.

No agreed "golden standard" exists for analysis of antioxidant capacity. The FRAP assay, which measures the total concentration of redox-active compounds (i.e. antioxidants), is easy, rapid to perform and require little plasma volume. The modified FRAP assay (31) measures water-soluble antioxidants (including uric acid, vitamin $\mathrm{C}$ and bilirubin) as well as most fat-soluble antioxidants. FRAP is regarded a reliable measurement of over-all ability to resist oxidative damage (30). In our present study of fetal antioxidant capacity, median FRAP concentration was actually higher in the infants of the preeclampsia group compared with controls, despite lower median gestational age than the control group. FRAP levels in both umbilical artery and vein correlated positively with previously analyzed (21) maternal FRAP levels (Pearson correlation 0.9, $p<0.001$ ). Uric acid concentration is elevated in maternal circulation in preeclampsia, which could influence maternal FRAP concentrations. We did not measure fetal uric acid concentrations in our study and are not aware of studies exploring this. It is therefore a limitation of this study that we are unaware of a potential effect on fetal FRAP by a possibly elevated uric acid concentration in infants of mothers with preeclampsia.

In this study of fetal oxidative stress, we limited the eligible study participants to those delivered by cesarean section without labor, to avoid the unpredictable oxidative stress resulting from various length and intensity of labor (39) and thereby a possible effect on the fetal (and maternal) concentrations of 8 -isoprostane or antioxidants.

Before this study, the SD for 8-isoprostane concentrations in umbilical artery and vein were not known. Assuming we wanted to detect at least one standardized difference between the groups, if present, we would need a total number of 30 patients to detect such a difference (with a standard significance level of 5\% and a power of 80\%) (40). Retrospectively, with mean 8-isoprostane in the umbilical artery (which may better reflect the fetal status than the umbilical vein) of 280 $\mathrm{pg} / \mathrm{mL}$, and a SD of $101 \mathrm{pg} / \mathrm{mL}$ for the control group, we could have detected a difference between the included preeclampsia and control group of $95 \mathrm{pg} / \mathrm{mL}$ (with a power of $80 \%$ and a significance level of 5\%), if present.

A limitation of our study is that preeclamptic patients and controls were not matched for gestational length, and the median gestational age in the preeclampsia group was lower than in the control group (238 versus $271 \mathrm{~d}$, Table 1). Obtaining umbilical cord blood from a group matching the premature preeclamptic pregnancies is difficult; cesarean section is not done in premature healthy pregnancies and cordocentesis would not be done for research purpose alone. Differences in gestational length between the study groups could potentially affect the conclusions. We did not find any correlation between gestational age and level of total 8-isoprostane in umbilical vein or artery in our whole study group, or in the preeclampsia group when analyzed separately. This is in contrast to a study by Comporti et al. (41), showing an inverse correlation between gestational age and concentrations of umbilical vein plasma F2-isoprostanes (measured as free isoprostanes only, not including phospholipid-bound isoprostanes), with higher levels in premature deliveries. The reason for premature delivery in their study was not stated.

In the paper originally describing the GC-MS method used in our study for measuring 8-isoprostane (29), the level of 8-isoprostane in adults was half of what was found in our group of healthy control infants. The median newborn level of $276 \mathrm{pg} / \mathrm{mL}$ in the umbilical artery would seem to be from a situation of "increased oxidative stress," if compared directly with adult values. We speculate that this higher level is "physiologic" for the newborn period, and could be a consequence of growth, development and increased metabolism in the fetus.

Intervention with antioxidants (vitamin $\mathrm{E}$ and $\mathrm{C}$ ) has been tried in a clinical study in women at high risk for developing preeclampsia, with effect on reducing the prevalence of preeclampsia. No improved outcome was found in the infants (35), but the study was not powered to test fetal outcome. The authors also demonstrated a reduced longitudinal elevation of the oxidative stress marker 8-isoprostane in the group receiving antioxidants (42). Other interventions, such as reducing blood pressure in preeclamptic women, do not improve outcome for the infants (43). Despite epidemiologic evidence that antioxidants could be beneficial in diseases such as atherosclerosis, similar positive effects in clinical intervention studies have not been demonstrated (44). Antioxidants could also have potential side effects. Vitamin E given to very low birth weight babies, at risk for getting oxygen related diseases, 
prevented retinopathy of prematurity, but was associated with an increased risk of sepsis (45). Multivitamins given to healthy infants was recently found to be associated with increased risk of asthma and food allergy (46).

In a recent study of breast-fed, full-term healthy infants, the concentration of F2-isoprostanes was found to be higher in infants than in adults (47). This was interpreted as augmented oxidative stress in infants, and antioxidant therapy was suggested. It has been said that "all studies of premature infants show that they are suffering from oxidative stress, but studies of antioxidants have showed no, minimal or controversial benefit" (26). In the light of this lack of demonstrated benefits and the possible harmful side effects of antioxidants, one has to evaluate carefully the short- and long-term effects on the infants of antioxidant intervention studies to prevent preeclampsia.

In conclusion, we found no difference in median fetal concentration of the oxidative stress marker 8-isoprostane between newborns of preeclamptic and control pregnancies, neither in umbilical cord vein nor artery. A previously demonstrated increased oxidative stress in the maternal circulation in preeclampsia is thus not reflected in the fetal circulation.

\section{REFERENCES}

1. Roberts JM, Redman CW 1993 Pre-eclampsia: more than pregnancy-induced hypertension. Lancet 341:1447-1451

2. Barden A, Beilin LJ, Ritchie J, Croft KD, Walters BN, Michael CA 1996 Plasma and urinary 8-iso-prostane as an indicator of lipid peroxidation in pre-eclampsia and normal pregnancy. Clin Sci (Lond) 91:711-718

3. Hubel CA 1999 Oxidative stress in the pathogenesis of preeclampsia. Proc Soc Exp Biol Med 222:222-235

4. Lee VM, Quinn PA, Jennings SC, Ng LL 2003 Neutrophil activation and production of reactive oxygen species in pre-eclampsia. J Hypertens 21:395-402

5. Sargent IL, Germain SJ, Sacks GP, Kumar S, Redman CW 2003 Trophoblast deportation and the maternal inflammatory response in pre-eclampsia. J Reprod Immunol 59:153-160

6. Maynard SE, Min JY, Merchan J, Lim KH, Li J, Mondal S, Libermann TA, Morgan JP, Sellke FW, Stillman IE, Epstein FH, Sukhatme VP, Karumanchi SA 2003 Excess placental soluble fms-like tyrosine kinase 1 (sFlt1) may contribute to endothelial dysfunction, hypertension, and proteinuria in preeclampsia. J Clin Invest 111:649-658

7. Bowen RS, Moodley J, Dutton MF, Theron AJ 2001 Oxidative stress in preeclampsia. Acta Obstet Gynecol Scand 80:719-725

8. Kato H, Yoneyama Y, Araki T 1997 Fetal plasma lipid peroxide levels in pregnancies complicated by preeclampsia. Gynecol Obstet Invest 43:158-161

9. Braekke K, Holthe MR, Harsem NK, Fagerhol MK, Staff AC 2005 Calprotectin, a marker of inflammation, is elevated in the maternal but not in the fetal circulation in preeclampsia. Am J Obstet Gynecol 193:227-233

10. Tsukahara H, Ohta N, Sato S, Hiraoka M, Shukunami K, Uchiyama M, Kawakami H, Sekine K, Mayumi M 2004 Concentrations of pentosidine, an advanced glycation end-product, in umbilical cord blood. Free Radic Res 38:691-695

11. Godfrey KM, Barker DJ Fetal nutrition and adult disease. 2000 Am J Clin Nutr 71:1344S-1352S

12. Tenhola S, Rahiala E, Martikainen A, Halonen P, Voutilainen R 2003 Blood pressure, serum lipids, fasting insulin, and adrenal hormones in 12-year-old children born with maternal preeclampsia. J Clin Endocrinol Metab 88:1217-1222

13. Vatten LJ, Romundstad PR, Holmen TL, Hsieh CC, Trichopoulos D, Stuver SO 2003 Intrauterine exposure to preeclampsia and adolescent blood pressure, body size, and age at menarche in female offspring. Obstet Gynecol 101:529-533

14. Innes KE, Byers TE 1999 Preeclampsia and breast cancer risk. Epidemiology 10:722-732

15. Ros HS, Lichtenstein P, Ekbom A, Cnattingius S 2001 Tall or short? Twenty years after preeclampsia exposure in utero: comparisons of final height, body mass index, waist-to-hip ratio, and age at menarche among women, exposed and unexposed to preeclampsia during fetal life. Pediatr Res 49:763-769

16. Roberts LJ, Morrow JD 2000 Measurement of F(2)-isoprostanes as an index of oxidative stress in vivo. Free Radic Biol Med 28:505-513

17. Davi G, Ciabattoni G, Consoli A, Mezzetti A, Falco A, Santarone S, Pennese E, Vitacolonna E, Bucciarelli T, Costantini F, Capani F, Patrono C 1999 In vivo formation of 8-iso-prostaglandin f2alpha and platelet activation in diabetes mellitus: effects of improved metabolic control and vitamin E supplementation. Circulation 99:224-229
18. Morrow JD 2004 Quantification of isoprostanes as indices of oxidant stress and the risk of atherosclerosis in humans. Arterioscler Thromb Vasc Biol 25:279-286

19. Walsh SW, Vaughan JE, Wang Y, Roberts LJ 2000 Placental isoprostane is significantly increased in preeclampsia. FASEB J 14:1289-1296

20. Staff AC, Halvorsen B, Ranheim T, Henriksen T 1999 Elevated level of free 8-iso-prostaglandin F2alpha in the decidua basalis of women with preeclampsia. Am J Obstet Gynecol 181:1211-1215

21. Harsem NK, Braekke K, Staff AC 2005 Augmented oxidative stress as well as antioxidant capacity in maternal circulation in preeclampsia. Eur J Obstet Gynecol Reprod Biol 128:209-215

22. Moretti M, Phillips M, Abouzeid A, Cataneo RN, Greenberg J 2004 Increased breath markers of oxidative stress in normal pregnancy and in preeclampsia. Am J Obstet Gynecol 190:1184-1190

23. Raijmakers MT, Dechend R, Poston L 2004 Oxidative stress and preeclampsia. rationale for antioxidant clinical trials. Hypertension 44:374-380

24. Rogers S, Witz G, Anwar M, Hiatt M, Hegyi T 2000 Antioxidant capacity and oxygen radical diseases in the preterm newborn. Arch Pediatr Adolesc Med 154:544-548

25. Saugstad OD 2003 Bronchopulmonary dysplasia-oxidative stress and antioxidants. Semin Neonatol 8:39-49

26. Thibeault DW 2000 The precarious antioxidant defenses of the preterm infant. Am J Perinatol 17:167-181

27. ACOG Committee on Obstetric Practice 2002 ACOG practice bulletin. Diagnosis and management of preeclampsia and eclampsia. Number 33, January 2002. American College of Obstetricians and Gynecologists. Int J Gynaecol Obstet 77:67-75

28. Skjaerven R, Gjessing HK, Bakketeig LS 2000 Birthweight by gestational age in Norway. Acta Obstet Gynecol Scand 79:440-449

29. Nourooz-Zadeh J, Gopaul NK, Barrow S, Mallet AI, Anggard EE 1995 Analysis of F2-isoprostanes as indicators of non-enzymatic lipid peroxidation in vivo by gas chromatography-mass spectrometry: development of a solid-phase extraction procedure. J Chromatogr B Biomed Appl 667:199-208

30. Benzie IF, Strain JJ 1996 The ferric reducing ability of plasma (FRAP) as a measure of "antioxidant power": the FRAP assay. Anal Biochem 239:70-76

31. Halvorsen BL, Holte K, Myhrstad MC, Barikmo I, Hvattum E, Remberg SF, Wold AB, Haffner K, Baugerod H, Andersen LF, Moskaug O, Jacobs DR Jr, Blomhoff R 2002 A systematic screening of total antioxidants in dietary plants. J Nutr 132:461471

32. Griffiths HR, Moller L, Bartosz G, Bast A, Bertoni-Freddari C, Collins A, Cooke M, Coolen S, Haenen G, Hoberg AM, Loft S, Lunec J, Olinski R, Parry J, Pompella A, Poulsen H, Verhagen H, Astley SB 2002 Biomarkers. Mol Aspects Med 23:101-208

33. Baydas G, Karatas F, Gursu MF, Bozkurt HA, Ilhan N, Yasar A, Canatan H 2002 Antioxidant vitamin levels in term and preterm infants and their relation to maternal vitamin status. Arch Med Res 33:276-280

34. Sanchez-Vera I, Bonet B, Viana M, Sanz C 2004 Relationship between alphatocopherol content in the different lipoprotein fractions in term pregnant women and in umbilical cord blood. Ann Nutr Metab 48:146-150

35. Chappell LC, Seed PT, Briley AL, Kelly FJ, Lee R, Hunt BJ, Parmar K, Bewley SJ, Shennan AH, Steer PJ, Poston L 1999 Effect of antioxidants on the occurrence of pre-eclampsia in women at increased risk: a randomised trial. Lancet 354:810-816

36. Mikhail MS, Anyaegbunam A, Garfinkel D, Palan PR, Basu J, Romney SL 1994 Preeclampsia and antioxidant nutrients: decreased plasma levels of reduced ascorbic acid, alpha-tocopherol, and beta-carotene in women with preeclampsia. Am J Obstet Gynecol 171:150-157

37. Schiff E, Friedman SA, Stampfer M, Kao L, Barrett PH, Sibai BM 1996 Dietary consumption and plasma concentrations of vitamin $\mathrm{E}$ in pregnancies complicated by preeclampsia. Am J Obstet Gynecol 175:1024-1028

38. Palan PR, Mikhail MS, Romney SL 2001 Placental and serum levels of carotenoids in preeclampsia. Obstet Gynecol 98:459-462

39. Rogers MS, Mongelli JM, Tsang KH, Wang CC, Law KP 1998 Lipid peroxidation in cord blood at birth: the effect of labour. Br J Obstet Gynaecol 105:739-744

40. Altman DG 1999 Clinical Trials. Practical Statistics for Medical Research. Chapman \& Hall, London, pp 440-476

41. Comporti M, Signorini C, Leoncini S, Buonocore G, Rossi V, Ciccoli L 2004 Plasma F2-isoprostanes are elevated in newborns and inversely correlated to gestational age. Free Radic Biol Med 37:724-732

42. Chappell LC, Seed PT, Kelly FJ, Briley A, Hunt BJ, Charnock-Jones DS, Mallet A, Poston L 2002 Vitamin C and E supplementation in women at risk of preeclampsia is associated with changes in indices of oxidative stress and placental function. Am J Obstet Gynecol 187:777-784

43. von Dadelszen P, Ornstein MP, Bull SB, Logan AG, Koren G, Magee LA 2000 Fall in mean arterial pressure and fetal growth restriction in pregnancy hypertension: a meta-analysis. Lancet 355:87-92

44. Stocker R, Keaney JF Jr 2004 Role of oxidative modifications in atherosclerosis. Physiol Rev 84:1381-1478

45. Brion LP, Bell EF, Raghuveer TS 2003 Vitamin E supplementation for prevention of morbidity and mortality in preterm infants. Cochrane Database Syst Rev CD003665

46. Milner JD, Stein DM, McCarter R, Moon RY 2004 Early infant multivitamin supplementation is associated with increased risk for food allergy and asthma. Pediatrics 114:27-32

47. Friel JK, Friesen RW, Harding SV, Roberts LJ 2004 Evidence of oxidative stress in full-term healthy infants. Pediatr Res 56:878-882 